\title{
INFLUENCIA DEL DISTANCIAMIENTO Y NÚMERO DE PLANTAS POR GOLPE EN EL RENDIMIENTO DE GRANO DE QUINUA (Chenopodium quinoa Willd)
}

\author{
INFLUENCE OF DISTANCING AND NUMBER OF PLANTS BY COUP IN \\ THE PRODUCTION OF QUINOA GRAIN (Chenopodium quinoa Willd)
}

\author{
${ }^{1}$ Nivardo Núñez Torreblanca, ${ }^{2}$ Magno Robles Tello, ${ }^{3}$ Marcos Alvarez Quispe
}

\begin{abstract}
RESUMEN
El presente trabajo de investigación "Influencia del distanciamiento y número de plantas por golpe en el rendimiento de grano de quinua (Chenopodium quinoa Willd.)" se desarrolló en el Centro Experimental "Los Pichones" de la Facultad de Ciencias Agropecuarias de la UNJBG, con el objetivo de estudiar los efectos de cuatro distanciamientos $(20,30,40$ y $50 \mathrm{~cm}$ ) entre golpes de siembra y número de plantas $(1,2,3, \mathrm{y} 4)$ por golpe de siembra en el rendimiento de grano; el genotipo empleado fue la variedad Salcedo INIA. Se utilizó un diseño experimental de bloques completos al azar, con disposición factorial de los tratamientos con tres repeticiones. Los resultados muestran que el factor distanciamiento entre golpe de siembra no tuvo efecto alguno sobre el número de panojas secundarias, peso de panojas secundarias, peso de grano de panojas secundarias, peso de panoja principal, peso de grano de panoja principal. El factor número de plantas en cambio sí afectó las variables evaluadas, la quinua respondió mostrando valores decrecientes cuando el número de plantas por golpe de siembra fue mayor a tres. El número óptimo de plantas por golpe de siembra fue tres, con las cuales se presentó el mayor rendimiento de grano de quinua: 3706,80 kilogramos por hectárea.
\end{abstract}

Palabras clave: Distanciamientos, número de plantas, quinua, rendimiento.

\begin{abstract}
This research "Influence of the distance and number of plants by blow in the production of grain of quinoa (Chenopodium quinoa Willd.)", was developed in the Experimental Center "Los Pichones" of the Faculty of Agricultural Sciences of the UNJBG. This was made with the objective of studying the effects of four spacings $(20,30,40$ and $50 \mathrm{~cm})$ between planting strokes and number of plants $(1,2,3$, and 4$)$ per sowing stroke in grain yield, the genotype employee was the Salcedo INIA variety; an experimental design of complete blocks was used at random, with a factorial disposition of the treatments with three repetitions. The results show that the distancing factor between planting strokes had no effect on the number of secondary panicles, weight of secondary panicles, grain weight of secondary panicles, weight of main panicle, weight of main panicle grain. The factor number of plants in change did affect the variables evaluated, quinoa responded by showing decreasing values when the number of plants per planting stroke was greater than three. The optimum number of plants per sowing stroke was three, with which the highest yield of quinoa grain was 3706,80 kilograms per hectare.
\end{abstract}

Keywords: Distances, number of plants, quinoa, yield.

\section{INTRODUCCIÓN}

La FAO (2011) considera que el valor nutricional de la quinua (Chenopodium quinoa Willd) es excelente, por cuanto variados estudios señalan que contiene entre 13,81 y $21,9 \%$ de proteínas de alta calidad que son del tipo globulina y albúmina; el contenido de aminoácidos que se encuentran en el núcleo del grano también le confieren alto valor alimenticio, a diferencia del trigo o arroz que los tienen en su exosperma; la quinua es señalada como el único alimento de origen vegetal capaz de proporcionar todos los aminoácidos esenciales, vitaminas, oligoelementos. Este preciado grano andino ha despertado el interés de muchos investigadores más allá de las fronteras de Sudamérica. Asimismo, García et al. (2017) indican que la calidad del grano de quinua para la alimentación

\footnotetext{
${ }^{1}$ Facultad de Ciencias Agropecuarias, Universidad Nacional Jorge Basadre Grohmann. Tacna-Perú. E-mail: ntor 01@hotmail.com ${ }^{2}$ Facultad de Ciencias Agropecuarias, Universidad Nacional Jorge Basadre Grohmann. Tacna-Perú. E-mail: pacarenka@hotmail.com

${ }^{3}$ Facultad de Ciencias Agropecuarias, Universidad Nacional Jorge Basadre Grohmann. Tacna-Perú. E-mail: marcos19a@hotmail.com 
humana, está en el valor nutricional y contenido de proteína $(18 \%)$, balance de aminoácidos, vitaminas, minerales y que se muestra libre de gluten. La quinua tiene buenas características que son aprovechadas para la alimentación humana, también se utiliza como materia prima para diferentes tipos de procesamiento de alimentos (Larico et al., 2014).

Si bien el cultivo de la quinua se ha adaptado en otros continentes, los países de la zona andina son los principales productores de grano a nivel mundial, alcanzando una producción de 70000 toneladas y de este total al Perú le corresponde 40000 toneladas; 28 000, a Bolivia; y en menor proporción a Ecuador. A nivel del continente europeo la producción es del orden de 210 toneladas (FAO, 2011).Un aspecto que influye de manera importante en el manejo de un cultivo es la población de plantas en el terreno de cultivo, se debe procurar que sea un número adecuado tanto para que las labores de cultivo se realicen de forma apropiada, así como para el control de plagas y enfermedades; especialmente para que las plantas puedan competir satisfactoriamente entre ellas sin que ocurran desequilibrios que afecten los rendimientos.

Específicamente en el caso de la quinua (Chenopodium quinoa Willd), se observa que en las condiciones de la costa de Tacna se utiliza inadecuadamente la cantidad de semilla, lo que genera un excesivo número de plantas por línea de siembra que conlleva a una serie de eventos como competencia entre las mismas plantas por elementos nutritivos, agua, luz, y espacio, principalmente; además genera un medio propicio para el ataque de plagas y enfermedades, lo que inevitablemente tendrá efectos negativos en el rendimiento de grano. La quinua regularmente se siembra en líneas a chorro continuo; sin embargo, en esas condiciones los productores de la zona no realizan ninguna labor para tener una población de plantas que no sea excesiva, lo que da lugar a plantas pequeñas con tallos delgados y panojas con poco desarrollo, que determinan pérdidas y rendimientos disminuidos. $\mathrm{El}$ presente estudio se propone analizar el efecto de la siembra en golpes con diferentes distanciamientos y número de plantas en el rendimiento de grano.

La quinua es considerada una especie capaz de resistir factores adversos como la sequía y salinidad del suelo que están presentes en la costa sur del Perú y en otras regiones del mundo. Su tolerancia a la sequía se atribuye en parte a caracteres morfológicos, como una raíz bastante ramificada y papilas higroscópicas con oxalato de calcio en la cutícula de la hoja, que le permite reducir los niveles de transpiración (Canahua, 1997); otro estudio conducido por Jensen et al. (2000) muestra que la quinua posee mecanismos que le permiten resistir la sequía debido a bajos potenciales osmóticos, una relación baja entre peso a plena turgencia y peso seco, baja elasticidad, capacidad para mantener turgencia positiva a bajos potenciales hídricos de la hoja. También se observó que la quinua es capaz de recuperarse rápidamente luego de un episodio de sequía, recupera con facilidad su nivel anterior de fotosíntesis y área foliar específica (Rasmussen, 1997).

El fruto de la quinua es un grano (aquenio) y aunque muchos consideren que se trata de un cereal, no lo es, tampoco una monocotiledónea. No obstante, esta confusión conduce comparar a los cereales con la quinua. En el caso de los primeros, estos manifiestan su rendimiento de grano más alto en correspondencia a densidades de población de plantas relativamente baja, en algunos casos por debajo de 200 plantas por metro cuadrado; de modo que cualquier incremento en la cantidad de semillas, con la pretensión de obtener los mayores rendimientos, solo genera mayores costos de producción (Hay y Walker, 1994).

En términos generales, el incremento de la población de plantas genera que el patrón de acumulación de biomasa se modifique notoriamente; lo cual provoca que el crecimiento lento inicial del grano, antes de alcanzar la gran fase de mediados del periodo, disminuya. En tal situación las plantas expresan una tasa de producción acorde con la intersección de la luz y el índice de área foliar, característicos de la comunidad a la que pertenecen. Al aglomerarse las plantas dentro de la misma comunidad, se establecen interacciones demasiado fuertes: se interfieren entre ellas por la luz; su demanda evaporativa es limitada; así como el área para la recepción de lluvia y $\mathrm{CO}_{2}$ y el volumen de suelo, que proporcionan la humedad almacenada y los nutrientes, son también menores (Loomis y Connor, 2002).

Las plantas, para soportar condiciones desfavorables, han desarrollado diversos mecanismos; entre los cuales se encuentra la plasticidad, que se entiende como una medida de la cantidad o proporción en que la expresión de las características morfológicas cambia por efecto de las variaciones del ambiente. La plasticidad de la forma se refiere al hecho de que el mismo genotipo puede producir diferentes formas en ambientes heterogéneos, por lo que juega un rol importante en el proceso de adaptación de plantas a su ambiente natural. Si el análisis de caracteres en dos ambientes diferentes da por resultado una baja correlación, se tiene un indicador de alta plasticidad que se observa en cambios en la estimación. (Reyes y Martínez, 2001).

La mayoría de los estudios sobre la quinua se han desarrollado en el Altiplano; Barahona (1975) realizó una investigación en condiciones de campo en el distrito de Yunguyo al sur de Puno, para estudiar el efecto del distanciamiento y densidad de siembra de variedades de quinua (Chenopodium quinoa Willd), utilizando tres factores de estudio: variedades, distanciamiento entre surcos y densidades de siembra. Dicha investigación llegó a la conclusión de que no hubo diferencias significativas entre los promedios de las densidades de siembra; sin embargo, los mayores rendimientos de grano se obtuvieron con la menor 
densidad ( $8 \mathrm{~kg}$ de semilla por hectárea). Además no encontró diferencias significativas entre distanciamientos, no obstante los mayores rendimientos promedio se presentaron con el distanciamiento de $45 \mathrm{~cm}$; tampoco se encontró diferencias estadísticas significativas para la interacción distanciamientos por densidades. Por otro lado, Espinoza (2016) en su estudio concluye que a mayor población de plantas por hectárea hay un aumento en el promedio de la rentabilidad, siendo la mayor rentabilidad promedio de $76 \%$ a la mayor densidad $\mathrm{D}_{2}(20 \mathrm{~cm})$, mientras que para la menor densidad $\mathrm{D}_{1}(25 \mathrm{~cm})$ la rentabilidad disminuye a $72 \%$.

Otra investigación en el Altiplano peruano se efectuó a 27 kilómetros al sur de Puno, con el objetivo de estudiar el efecto de dosis de nitrógeno y densidad de siembra en quinua (Chenopodium quinoa Willd); allíse consideraron dos factores: a) densidad de siembra de 8 , 10,12 y 14 kilogramos de semilla por hectárea, b) dosis de nitrógeno en un rango de 00 hasta 160 kilogramos por hectárea. La conclusión fue que no se presentaron diferencias estadísticas para el rendimiento de grano de quinua, nitrógeno, densidad de siembra, ni para interacción densidad por nitrógeno (Meza, 1976).

Montenegro y Coral (1984) - con el objetivo de conocer diferentes densidades de siembra, que variaron entre 100 a 500 mil plantas/ha sobre la altura y producción de plantas de quinua de la variedad Sajama - demostraron que al aumentar la densidad de siembra, tanto la altura como la producción dejaron de ser óptimas debido a la competencia entre las plantas, y llegaron a la conclusión de que la mejor densidad de siembra fue de $250 \mathrm{mil}$ plantas/ha para las condiciones de Torobajo en Colombia.La influencia del número de plantas por unidad de área en relación a otras características fue estudiada por Quispe (1981), quien analizó los factores influencia de luz y densidad de siembra en la expresión del período vegetativo de dos variedades de quinua (Chenopodium quinoa Willd).

Aquel estudio se realizó en condiciones de invernadero en la Universidad Nacional del Altiplano de Puno y se encontró que hasta 40 plantas por maceta reducen la altura de la planta, la longitud de panoja y el diámetro del tallo; pero prolonga los días de la floración y hay mayor producción de grano. El factor luz actúa en forma independiente de los factores variedad y densidad en la variación de altura de planta, longitud de panoja, diámetro de tallo, días de la floración y maduración. La interacción variedad por densidad es notoria en todas estas características.

Algunos estudios con relación a densidades de siembra que definen el número de plantas en campo también se dieron en Ecuador. Calle y Salamea en 1982, estudiaron dos sistemas de siembra con cuatro densidades en dos ecotipos de quinua (Chenopdium quinoa Willd) en Tumbaco Pichincha (Ecuador), y concluyeron que en el sistema de siembra en líneas se presentó la mayor altura de planta $(1,45 \mathrm{~m})$ y en el sistema a voleo la menor altura de planta $(1.31 \mathrm{~m})$; asimismo este sistema permitió el rendimiento de grano más elevado con una densidad de 12 kilogramos de semilla por hectárea, además encontraron correlación entre el rendimiento de grano y la altura de la planta.

E1 estudio de 1a densidad de plantas ha sido considerado importante en otros cultivos andinos como Amarantus spp. En el que se investigó las respuestas al espaciamiento entre hileras y la densidad de plantas; para esto se utilizaron los cultivares de amaranto K283, K 343, K 432 y MT - 3, en dos localidades norteamericanas. Las poblaciones de plantas establecidas fueron 74000,173000 y 272000 plantas por hectárea con un espaciamiento entre surcos de 30 y $76 \mathrm{~cm}$. La evaluación de las respuestas muestran que en la primera y segunda localidad (Prosper, Williston) se observó un rendimiento superior a $12 \%$ en la población más baja en comparación con la más alta en la primera campaña, sin que ocurriera similar respuesta en la segunda campaña. Otro resultado relevante fue que en el distanciamiento entre surcos no resultó significativo el espaciamiento de $76 \mathrm{~cm}$ en ambas localidades; contrario al resultado del espaciamiento entre surcos de $30 \mathrm{~cm}$ que sí fue significativo; los cultivos de Amarantus cruentus $L$. produjeron más grano que los de Amarantus hypochondricus L. (Henderson et al., 2000).

\section{MATERIALES Y MÉTODOS}

Esta investigación se ejecutó en el Centro Experimental "Los Pichones" de la Escuela Profesional de Agronomía de la Universidad Nacional Jorge Basadre Grohmann de Tacna, a $550 \mathrm{msnm}$. Para el estudio se utilizó semilla certificada de la variedad Salcedo INIA, procedente de Puno.

El diseño experimental utilizado fue de bloques completos al azar con disposición factorial, con dos factores en estudio: a) cuatro distanciamientos entre golpes, b) cuatro números de plantas por golpe de siembra, configurando un total de 16 tratamientos y tres repeticiones.

Los factores en estudio fueron factor A: distanciamientos entre golpes de siembra, con cuatro niveles $(20,30,40$ y $50 \mathrm{~cm})$, y el factor $\mathrm{B}$ : número de plantas por golpe de siembra, con cuatro niveles $(1,2$, 3 y 4 plantas). La distribución de los tratamientos se efectuó antes de la siembra, para lo cual se marcaron los distanciamientos entre golpes de siembra, se hizo el hoyado correspondiente donde se colocaron los fertilizantes cubriéndolos con una capa de tierra; luego, según el croquis de la distribución de tratamientos, se depositaron las semillas de quinua colocando un exceso de tres a cuatro semillas para todos los casos a fin de garantizar la germinación y tener el número de plantas planeado en el experimento. La fórmula de fertilización utilizada fue de 200 - 120 80 de $\mathrm{N}-\mathrm{P} 2 \mathrm{O} 5-\mathrm{K} 2 \mathrm{O}$. 


\section{RESULTADOS}

\section{Número de panojas secundarias por planta}

El análisis de variancia de número de panojas secundarias por planta de quinua por unidad experimental (tabla 1) muestra que la fuente de variabilidad repeticiones resultó con alta significancia estadística, lo cual indica que los bloques no se comportaron homogéneamente. El factor distanciamientos resultó no significativo, por lo tanto no afectó el número de panojas secundarias; a diferencia del anterior, el factor número de plantas por golpe presentó diferencias estadísticas altamente significativas, por ello se puede señalar que influyó en el número de panojas secundarias por planta de quinua. Para la interacción distanciamientos por número de plantas por golpe no hubo significación estadística, por ende, ambos factores actuaron de manera independiente uno del otro; con lo que toma importancia el factor principal número de plantas por golpe de siembra.

$\mathrm{El}$ análisis de variancia de regresión de número de panojas secundarias de quinua para número de plantas por golpe de siembra (tabla 2) resultó altamente significativo, lo que confirma que el número de plantas influyó significativamente en el número de panojas secundarias; sin embargo, el coeficiente de determinación indica que el $28,17 \%$ de la variabilidad en el número de panojas secundarias se debe al número de plantas por golpe de siembra. La ecuación resultante es la siguiente: $\mathrm{Y}=14,80333-1,11450 \mathrm{P}$.

En la figura 1 se observa el efecto del número de plantas por golpe de siembra. Esto indica que el número de panojas secundarias disminuye en función del número de plantas por golpe; de este modo cuando en el golpe de siembra hubo una planta, el número de panojas secundarias fue de 13,69 , para luego disminuir progresivamente; así el promedio disminuye hasta 10,35 panojas secundarias cuando en el golpe de siembra crecieron cuatro plantas.

\section{Peso de panoja secundaria por planta}

$\mathrm{El}$ análisis de variancia de peso de panojas secundarias en cada planta (tabla 1) muestra que para la fuente de variabilidad repeticiones no se presentaron diferencias estadísticas, indicando que los bloques fueron homogéneos. El factor distanciamientos resultó no significativo, por lo tanto no afectó el peso de panojas secundarias; a diferencia del anterior, el factor número de plantas por golpe presentó diferencias estadísticas altamente significativas, con ello se puede señalar que influyó en el peso de panojas secundarias por planta; para la interacción distanciamientos por número de plantas por golpe no hubo significación estadística, con lo cual ambos factores actuaron de manera independiente uno del otro; en consecuencia, toma importancia el factor principal número de plantas por golpe de siembra.
El análisis de variancia de regresión de peso de panojas secundarias de quinua para número de plantas por golpe de siembra (tabla 2) resultó altamente significativo, lo que confirma que el número de plantas influyó significativamente en el peso de panojas secundarias, el coeficiente de determinación indica que el $44,73 \%$ de la variabilidad en el peso de panojas secundarias se debe al número de plantas por golpe de siembra. La ecuación resultante es la siguiente: $\mathrm{Y}=$ 151,09875-20,77567P.

En la figura 2, se presenta gráficamente el efecto del número de plantas por golpe de siembra sobre el peso de panojas secundarias de plantas de quinua, lo que indica que el peso de panojas secundarias disminuye en función del número de plantas por golpe; de este modo cuando en el golpe de siembra hubo una planta, el peso de panojas secundarias fue de 130,32 gramos, para disminuir después progresivamente, así los valores descienden hasta un promedio de 68 gramos cuando en el golpe de siembra crecieron cuatro plantas.

\section{Peso de grano de panoja secundaria por planta}

El análisis estadístico de peso de grano de panojas secundarias por planta de quinua (tabla 1 ) indica que en la fuente de variabilidad repeticiones no se presentaron diferencias estadísticas, aceptándose la homogeneidad de bloques. El factor distanciamientos resultó no significativo, consecuentemente no afectó el peso de grano de las panojas secundarias; a diferencia del anterior, el factor número de plantas por golpe presentó diferencias estadísticas altamente significativas, por lo que se puede señalar que influyó en el peso de grano de las panojas secundarias de quinua; para la interacción distanciamientos por número de plantas por golpe no hubo significación estadística, en consecuencia, ambos factores actuaron de manera independiente uno del otro; por ello adquiere importancia el factor principal número de plantas por golpe de siembra.

El análisis de variancia de regresión de peso de grano de panojas secundarias de quinua para número de plantas por golpe de siembra (tabla 2) resultó altamente significativo, lo cual confirma que el número de plantas influyó significativamente en el peso de panojas secundarias, el coeficiente de determinación indica que el $54,34 \%$ de la variabilidad en el peso de panojas secundarias se debe al número de plantas por golpe de siembra. La ecuación resultante es la siguiente: $\mathrm{Y}=$ $54,49958-7,71842 \mathrm{P}$.

En la figura 3 se observa el efecto del número de plantas por golpe de siembra sobre el peso de grano de panojas secundarias de plantas de quinua, lo cual indica que el peso de grano de panojas secundarias disminuye en función del número de plantas por golpe; de este modo cuando en el golpe de siembra hubo una planta, el peso de panojas secundarias fue de 46,78 gramos, para ir disminuyendo progresivamente, así los valores descendieron hasta un promedio de 23,63 gramos cuando en el golpe de siembra crecieron cuatro plantas. 
Tabla 1. ANVA de las variables: número de panojas secundarias (NPS), peso de grano de panoja secundaria (PPS), peso de grano de panoja secundaria porplanta (PGPS).

\begin{tabular}{lllll}
\hline F de V & gl & NPS & PPS & PGPS \\
\hline Repeticiones & 2 & $28,63^{* *}$ & $648,82 \mathrm{~ns}$ & $16,75 \mathrm{~ns}$ \\
Distanciamientos & 3 & $2,37 \mathrm{~ns}$ & $1319,35 \mathrm{~ns}$ & $133,32 \mathrm{~ns}$ \\
Núm. De plantas & 3 & $26,78^{* *}$ & $9540,10^{* *}$ & $1378,61^{* *}$ \\
Interacción & 9 & $2,67 \mathrm{~ns}$ & $527,57 \mathrm{~ns}$ & $43,13 \mathrm{~ns}$ \\
Error Exp. & 30 & 3,19 & 642,43 & 54,00 \\
Total & 47 & & & \\
CV & & $14,87 \%$ & $25,56 \%$ & $20,80 \%$ \\
\hline
\end{tabular}

$\mathrm{CV}=$ coeficiente de variación, ${ }^{* *}=$ Significativo, $\mathrm{NS}=$ no significativo.

Tabla2. Análisis de regresión de las variables: número de panojas secundarias (NPS), peso de grano de panoja secundaria (PPS), peso de grano de panoja secundaria por planta (PGPS).

\begin{tabular}{lllll}
\hline F de V & gl & NPS & PPS & PGPS \\
\hline Regresión & 1 & $74,53 * *$ & 25898,00 & 3574,44 \\
Error exp. & 46 & 4,13 & 695,63 & 65,28 \\
Total & 47 & & & \\
$\mathrm{R}^{2}$ & & $28,17 \%$ & $44,73 \%$ & $54,34 \%$ \\
\hline
\end{tabular}

$\mathrm{F}$ de $\mathrm{V}=$ fuentes de variación, $\mathrm{gl}=$ grados de libertad, $\mathrm{R}^{2}=$ coeficiente de determinación, NPS $=$ número de panojas secundarias, $\mathrm{PPS}=$ peso de panoja secundaria; PGPS= peso de grano de panoja secundaria.

Fuente: Elaboración propia.

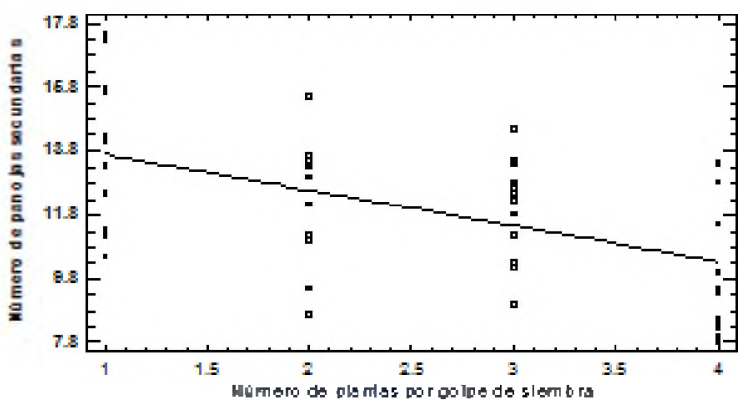

Figura 1. Regresión lineal del número de panojas secundarias por planta con respecto al número de plantas por golpe de siembra.

Fuente: Elaboración propia.

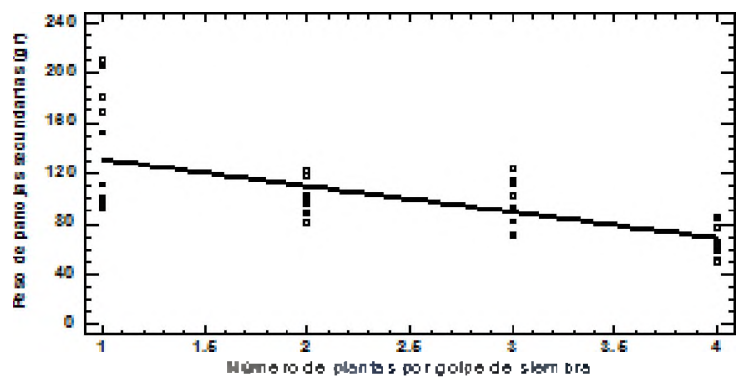

Figura 2. Regresión lineal de peso de panojas secundarias con respecto al número de plantas por golpe de siembra.

Fuente: Elaboración propia.

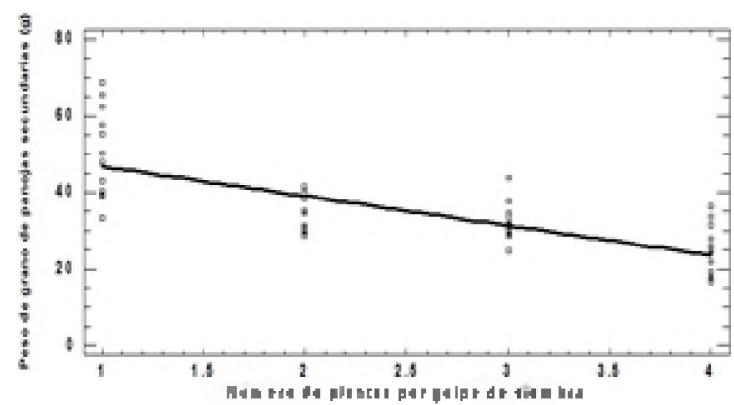

Figura 3. Regresión lineal de peso de grano de panojas secundarias con respecto al número de plantas por golpe de siembra.

Fuente: Elaboración propia.

\section{Peso de panoja principal por planta}

El análisis estadístico de peso de panoja principal por planta de quinua (tabla 3 ) evidencia que para la fuente de variabilidad repeticiones se presentaron diferencias estadísticas, indicando que los bloques no fueron homogéneos. El factor distanciamientos resultó no significativo, por lo tanto no afectó el peso de las panojas principales; a diferencia del anterior, el factor número de plantas por golpe presentó diferencias estadísticas altamente significativas, por lo que se puede señalar que influyó en el peso de las panojas principales de quinua; para la interacción distanciamientos por número de plantas por golpe no hubo significación estadística. Por ende, ambos factores actuaron de manera independiente uno del otro; por ello toma importancia el factor principal número de plantas por golpe de siembra.

El análisis de variancia de regresión de peso de panoja principal de quinua para número de plantas por golpe de siembra (tabla 4) resultó altamente significativo, lo que confirma que el número de plantas influyó significativamente en el peso de panoja principal, el coeficiente de determinación indica que el $45,86 \%$ de la variabilidad en el peso de panoja principal se debe al número de plantas por golpe de siembra. La ecuación resultante es la siguiente: $Y=82,28500-9,74825 \mathrm{P}$.

En la figura 4 se observa el efecto del número de plantas por golpe de siembra sobre el peso de panoja principal de plantas de quinua, lo cual indica que el peso de panoja principal disminuye en función del número de plantas por golpe; de este modo cuando en el golpe de siembra hubo una planta, el peso promedio de panoja principal fue de 72,54 gramos, para luego disminuir progresivamente, así los valores descendieron hasta un promedio de 43,29 gramos cuando en el golpe de siembra crecieron cuatro plantas.

\section{Peso de grano de panoja principal}

El análisis de variancia de peso de grano de panoja principal por planta de quinua (tabla 3) muestra que para la fuente de variabilidad repeticiones se presentaron diferencias estadísticas, indicando que los bloques no fueron homogéneos. El factor distanciamientos resultó no significativo, por lo tanto no afectó el peso de grano de las panojas principales; a 
diferencia del anterior, el factor número de plantas por golpe presentó diferencias estadísticas altamente significativas, por lo cual se puede señalar que influyó en el peso de las panojas principales de quinua; para la interacción distanciamientos por número de plantas por golpe no hubo significación estadística, en consecuencia ambos factores actuaron de manera independiente uno del otro; por ello toma importancia el factor principal número de plantas por golpe de siembra.

El análisis de variancia de regresión de peso de panoja principal de quinua para número de plantas por golpe de siembra (tabla 4) resultó altamente significativo, lo que confirma que el número de plantas influyó significativamente en el peso de grano de la panoja principal, el coeficiente de determinación indica que el $44,75 \%$ de la variabilidad en el peso de grano de la panoja principal se debe al número de plantas por golpe de siembra. La ecuación resultante es la siguiente: $\mathrm{Y}=$ $36,88750-4,34075 \mathrm{P}$.

En la figura 5 se observa el efecto del número de plantas por golpe de siembra sobre el peso de grano de la panoja principal de plantas de quinua, esto indica que el peso de panoja principal disminuye en función del número de plantas por golpe; de este modo cuando en el golpe de siembra hubo una planta, el peso promedio de panoja principal fue de 32,55 gramos, para disminuir progresivamente hasta un promedio de 19,52 gramos cuando en el golpe de siembra crecieron cuatro plantas.

\section{Rendimiento total de grano}

$\mathrm{El}$ análisis de variancia de rendimiento total de grano por hectárea (tabla 3) muestra que para la fuente de variabilidad repeticiones no se presentaron diferencias estadísticas, indicando que los bloques fueron homogéneos. El factor distanciamientos resultó no significativo, por lo cual se acepta que no afectó el rendimiento total de grano de quinua variedad Salcedo INIA; a diferencia del anterior, el factor número de plantas por golpe expresó diferencias estadísticas altamente significativas, por consiguiente, se puede aseverar que influyó en el rendimiento total de grano de quinua; la interacción distanciamientos por número de plantas por golpe no presentó significación estadística pues ambos factores actuaron de manera independiente uno del otro; por ello toma importancia el factor principal número de plantas por golpe de siembra.

$\mathrm{El}$ análisis de variancia de regresión de rendimiento total de grano de quinua por hectárea para número de plantas por golpe de siembra (tabla 4) resultó altamente significativo, lo que confirma que el número de plantas influyó significativamente en el rendimiento total de grano de la variedad Salcedo INIA, el coeficiente de determinación indica que el $40,92 \%$ de la variabilidad del rendimiento total obedece al número de plantas por golpe de siembra. La ecuación resultante es la siguiente: $\mathrm{Y}=550,48833+2206,95367 \mathrm{P}-$ $384,94833 \mathrm{P}^{2}$.
En la figura 6 se observa el efecto del número de plantas por golpe de siembra sobre el rendimiento total de grano de quinua variedad Salcedo INIA, esto indica que el rendimiento total de grano por hectárea se incrementa en función del número de plantas por golpe, hasta alcanzar un valor óptimo de 3 plantas por golpe de siembra con el cual se obtiene un rendimiento máximo de 3706,80 kilogramos por hectárea, para disminuir progresivamente hasta un promedio de 3 219,12 kilogramos por hectárea cuando en el golpe de siembra crecieron cuatro plantas.

Tabla 3 ANVA de las variables: peso de panoja principal (PPP), peso de grano de panoja principal por planta de quinua (PGPP), rendimiento total de grano de quinua $R T G$ ).

\begin{tabular}{lllll}
\hline F de V & gl & PPP & PGPP & RTG \\
\hline Repeticiones & 2 & $897,32^{* *}$ & $183,90^{* *}$ & $337343,52 \mathrm{~ns}$ \\
Distanciamientos & 3 & $270,73 \mathrm{~ns}$ & $54,07 \mathrm{~ns}$ & $356863,58 \mathrm{~ns}$ \\
Núm. De plantas & 3 & $2106,88^{* *}$ & $426,26^{* *}$ & $4334521,39^{* *}$ \\
Interacción & 9 & $69,14 \mathrm{~ns}$ & $\mathbf{1 4 , 9 5 \mathrm { ns }}$ & $548451,74 \mathrm{~ns}$ \\
Error Exp. & 30 & 96,07 & 19,44 & 312410,96 \\
Total & 47 & & & \\
CV & & $16,92 \%$ & $16,93 \%$ & $17,57 \%$ \\
\hline
\end{tabular}

$\mathrm{F}$ de $\mathrm{V}=$ Fuentes de variación, $\mathrm{gl}=$ Grados de libertad, PPP $=$ Peso de panoja principal, $\mathrm{PGPP}=$ Peso de grano panoja principal, $\mathrm{RTG}=$ Rendimiento total de grano, $\mathrm{CV}=$ coeficiente de variación, $* *=$ Significativo, $\mathrm{NS}=$ no significativo.

Fuente: Elaboración propia.

Tabla4. Análisis de regresión de las variables: peso de panoja principal (PPP), peso de grano de panoja principal por planta (PGPP), rendimiento total de grano de quinua $(R G T)$.

\begin{tabular}{lcccc}
\hline F de V & gl & PPP & PGPP & RTG \\
\hline Regresión & 1 & $5701,70^{* *}$ & $1130,53^{* * *}$ & $3574,44^{* *}$ \\
Error exp. & 46 & 146,30 & 30,35 & 65,28 \\
Total & 47 & & & \\
$\mathbf{R}^{2}$ & & $45,86 \%$ & $44,75 \%$ & $40,92 \%$ \\
\hline
\end{tabular}

$\mathrm{F}$ de $\mathrm{V}=$ fuentes de variación, $\mathrm{gl}=$ grados de libertad, $\mathrm{R}^{2}=$ coeficiente de determinación, $\mathrm{PPP}=$ Peso de panoja principal, PGPP $=$ Peso de grano panoja principal, $\mathrm{RTG}=$ Rendimiento total de grano .

Fuente: Elaboración propia.

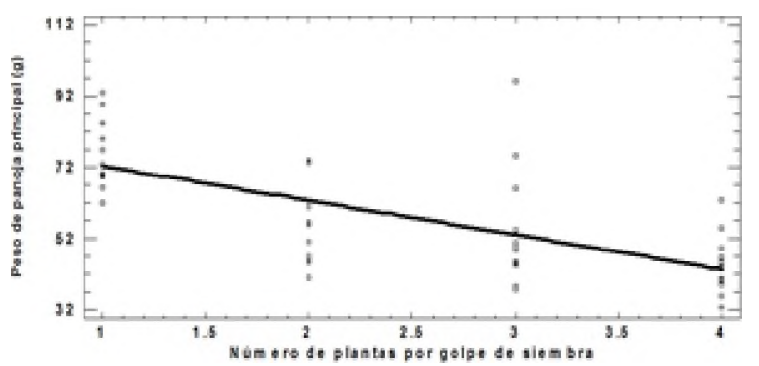

Figura 4. Regresión lineal de peso de panoja principal con respecto al número de plantas por golpe de siembra.

Fuente: Elaboración propia. 


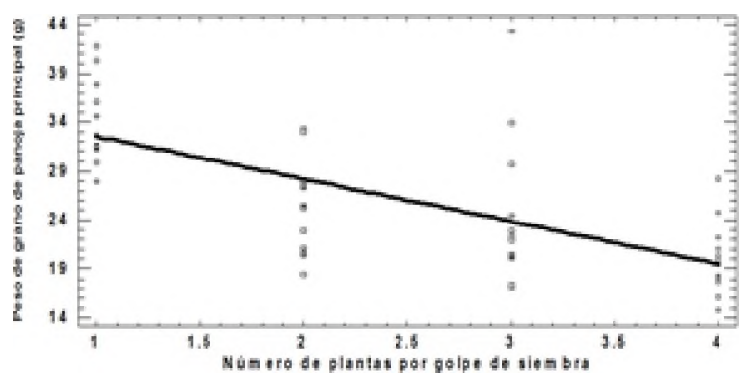

Figura 5. Regresión lineal de peso de grano de panoja principal con respecto al número de plantas por golpe de siembra.

Fuente: Elaboración propia .

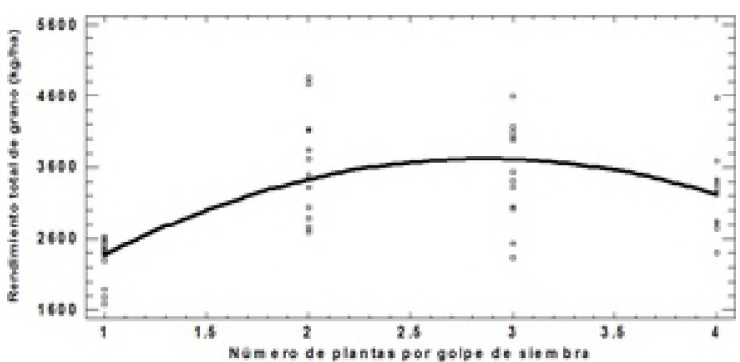

Figura 6. Regresión polinómica de rendimiento total de grano de quinua en relación al número de plantas por golpe de siembra.

Fuente: Elaboración propia.

\section{DISCUSIÓN}

Los resultados alcanzados en el presente estudio demostraron que los distanciamientos entre golpes de siembra no influyeron en el número de panojas secundarias, peso de grano de panojas secundarias, peso de panoja principal, peso de grano de panoja principal ni en el rendimiento total de grano por hectárea de la variedad de quinua Salcedo INIA. Sin embargo el número de plantas por golpe de siembra sí afectó de manera diferenciada las variables evaluadas, así se observó que en el análisis se dio una tendencia al decrecimiento en los valores de número de panojas secundarias, peso de panojas secundarias y su peso de grano, peso de panoja principal y su peso de grano que dieron por resultado cifras inferiores en golpes de siembra con mayor número de plantas. Estas respuestas se obtuvieron como resultado del análisis de plantas individuales, indicando que a medida que incrementa el número de plantas en un golpe de siembra se presentaron valores decrecientes, probablemente debido a la competencia entre plantas del mismo golpe de siembra.

Con relación al rendimiento total de grano por hectárea, este se caracterizó por una respuesta diferente cuya regresión fue de tipo polinómico; de manera que dentro del rango considerado en el experimento, el mayor número de plantas permisibles por golpe de siembra fue de tres, de acuerdo a estos resultados un número menor o mayor de plantas por golpe de siembra tiene efectos contrarios para los rendimientos. El rendimiento de 3706,80 kilogramos por hectárea con tres plantas por golpe puede considerarse aceptable.

Por otra parte los resultados muestran que, la siembra de quinua por golpes puede constituir una alternativa viable para la utilización racional de la semilla, lo que disminuiría de esta manera las cantidades que se emplean para siembras a chorro continuo. En lo referente a respuestas como el número de panojas secundarias los resultados encontrados indican que a medida que aumenta el número de plantas por golpe de siembra estas disminuyen, lo que concuerda con lo expresado por Mujica et al. (2000) quienes observaron que esta característica también varía con la población de plantas por unidad de área; esta variación también estaría relacionada con la plasticidad morfológica: concepto que se relaciona con la capacidad de los genotipos para expresar diferentes formas en ambientes heterogéneos (Reyes y Martínez, 2001).

El mayor rendimiento de grano de la quinua variedad Salcedo INIA se registró cuando en un golpe de siembra crecieron tres plantas (los distanciamientos entre golpes no influyeron), respuesta que se explicaría por la dinámica de poblaciones de cultivos que considera el número de individuos y sus características (Villalobos et al., 2002). Un número mayor a tres plantas por golpe de siembra tiene efectos contrarios sobre el rendimiento de grano debido a la existencia de interacciones e interferencias por luz, demanda evaporativa, recepción de luz y $\mathrm{CO} 2$, volumen de suelo para la obtención de agua y nutrientes (Loomis y Connor, 2002).

\section{CONCLUSIONES}

El distanciamiento entre golpes de siembra no influyó en las variables analizadas. A mayor número de plantas por golpe de siembra, decrece el número de panojas secundarias, peso de panojas secundarias, peso de panoja principal y sus respectivos pesos de grano.

El número óptimo de plantas por golpe de siembra para la variedad Salcedo INIA fue de tres, con el cual se obtuvo un rendimiento máximo de grano de quinua de 3706,80 kilogramos por hectárea.

\section{REFERENCIAS BIBLIOGRÁFICAS}

\section{Barahona, E. (1975). Efecto del distanciamiento densidad de siembra y variedades en el cultivo de la quinua (Chenopodium quinoa Willd). Puno, Perú. Resúmenes de investigaciones en quinua (Chenopodium quinoa Willd.) de la Universidad Nacional del Altiplano.}

Calle, T., y Salamea, H. (1982). Dos sistemas de siembra con cuatro densidades en dos 
ecotipos de quinua (Chenopodium quinoa Willd). Tesis (Título Ing. Agrónomo). Universidad Central del Ecuador. Quito, Ecuador.

Canahua, A. (1977). Observaciones del comportamiento de la quinua a la sequía. En: Actas del primer Congreso Internacional de Cultivos Andinos. Ayacucho, Perú, 390-392.

Espinoza, E. (2016). Adaptación del cultivo de quinua (Chenopodium quinoa willd) al cambio climático en los Andes del Perú. Revista del Instituto de Investigación (RIIGEO), FIGMMG-UNMSM, 9 (37), 15-23.

FAO. (2011). La quinua: Cultivo milenario para contribuir a la seguridad alimentaria mundial. Roma, Italia: Oficina Regional para América Latina y el Caribe.

García, M., Molano, J., Melo, D., y Deaquiz, Y. (2017). Quinua respuesta agronómica de la (Chenopodium quinoa Willd) Variedad dulce de Soracá a la fertilización en Ventaquemada-Boyacá. Rev. Cultura Científica, 5(15), 66-77.

Hay, Robert H., y Walker, A. (1994). An introduction to the physiology of crop yield. Copublished in the United States Wilth John Willy \& Sons. New York, USA: Longman Scientific \& Tecntechnical.

Henderson, T., Johnson, B. y Schneiter, A. (2000). Row Spacing, Plant Population, and Cultivar Effects on Grain Amaranth in the Northern Great Plains. Rev. Agronomy Journal Abstract - NEW CROP, 92 (2), 329-336.

Jensen, C., Jacobsen, S., Andersen, M., Núñez, N., Andersen, S., Rasmussen, L., \& Mogensen, V. (2000). Leaf gas exchange and water relation characteristics of field quinoa (Chenopodium quinoa Willd.) during soil drying. Rev. European Journal of Agronomy, (13) 1, 11-25.

Larico, J., Astete F. A., y Tacora, R. L. (2014). Perfil de ácidos grasos en granos tres cultivares de quinua (Chenopodium quinoa Willd) Sometidos a tres tipos de procesamiento. Rev. Investig. Altoandin, 16(1), 13 - 20.

Loomis, R., y Connor, J. (2002). Ecología de cultivos. Productividad y manejo en sistemas agrarios. Madrid, España: MundiPrensa.

Meza, A. (1976). Efecto de dosis de nitrógeno y densidad de siembra en quinua (Chenopodium quinoa Willd.) Var. Sajama. Puno, Perú. Resúmenes de investigaciones en quinua (Chenopodium quinoa Willd) de la
Universidad Nacional del Altiplano.

Montenegro, V., y Coral, E. (1984). Influencia de diferentes distancias de siembra sobre la altura y el rendimiento en el cultivo de quinua (Chenopodium quinoa Willd). Rev. Ciencias Agrícolas, Vol. 8, 103-107.

Mujica, A., Izquierdo, J., y Marathee, J. (2000). Origen $y$ descripción de la quinua (Chenopodium quinoa Willd). Ancestral cultivo andino, alimento del presente y futuro. FAO, Santiago, Chile: Universidad Nacional del Altiplano, Puno, Perú.

Quispe, A. (1981). Influencia de luz y densidad de siembra en el periodo vegetativo de dos variedades de quinua (Chenopodium quinoa Willd). Puno, Perú: Resúmenes de investigaciones en quinua (Chenopodium quinoa Willd) de la Universidad Nacional delAltiplano.

Rasmussen, L. (1977). Photosynthesis a quinoa (Chenopodium quinoa Willd.) $i$ relation til vand og kvaelstof. (Thesis of Master Science). Dep. Of Agricultural Sciencies. The Royal Veterinary \& Agricultura University, Copenhagen, Denmark.

Reyes, J., y Martínez, M. (2001). La plasticidad de las plantas. Universidad Autónoma de Puebla. Rev. Elementos. 8(41), 39-43.

Villalobos, F., Mateos, L., Orgaz, F., y Fereres, E. (2002). Fitotecnia: Bases y tecnologias de la producción agrícola. Madrid, España: Mundi-Prensa. 